\title{
Homocysteine, a Biomarker of Cardiovascular Diseases, in Psoriasis-A Case Control Study
}

\author{
Garg P1, Gupta S2*, Gupta N33 and Gupta N $^{4}$ \\ ${ }^{1}$ Consultant Dermatologist, Gupta Skin Clinic, India \\ ${ }^{2}$ Department of Dermatology, Venereology and Leprology, Maharaja Agrasen Medical \\ College, India \\ ${ }^{3}$ Department of Dermatology, Venereology and Leprology, SGT Medical College,
} Hospital \& Research Institute, India

${ }^{4}$ Department of Medicine, Ganga Ram Institute of Postgraduate Medical Education and Research (Sir Ganga Ram Hospital), India

*Corresponding author: Surinder Gupta, Associate Professor, Department of Dermatology, Venereology and Leprology, Maharaja Agrasen Medical College, Agroha, H.No. 829, Rishi Nagar, Hisar, Haryana, India-125001, Tel: 9812045055; Email: guptaskin@yahoo.com

\section{Abstract}

For atherosclerotic and cardiovascular diseases, stroke and peripheral arterial disease, hyperhomocysteinemia has been seen to be an independent marker. Psoriasis being a chronic inflammatory disease is associated with increased incidence of cardiovascular events. Hyperhomocysteinemia in psoriasis plays an important role in these events. Our study aims to investigate serum homocysteine levels in patients of psoriasis. Thirty patients of different severity of psoriasis (calculated with body surface area (BSA) and Psoriasis Area and Severity Index (PASI) score) were investigated for serum levels of homocysteine. Thirty controls were also studied for the same investigations. Serum homocysteine levels were significantly higher in psoriasis patients compared to control group $(\mathrm{p}<0.05)$. This may explain the excess cardiovascular morbidity and mortality in psoriasis.

Keywords: Homocysteine; Psoriasis; Cardiovascular disease

Abbreviations: PASI: Psoriasis Area and Severity Index; CVD: Cardiovascular Diseases; CRP: C-reactive protein; IL: interleukins; MAMC: Maharaja Agrasen Medical College; PASI: Psoriasis Area Severity Index; BSA: Body Surface Area; CLIA: Chemiluminescent Immunoassay; NAFLD: Non-alcoholic fatty liver disease.

\section{Introduction}

Psoriasis is a recurrent and chronic inflammatory disease with hyperproliferation of epidermal cells and affects 1$5 \%$ of population [1]. It is a multifactorial disease which includes genetic, environmental and immunological ( $\mathrm{T}$ cell mediated). 
Psoriasis has been studied, in recent years, for its association with metabolic disorder and it has been shown that psoriasis patients have increased incidence of cardiovascular diseases (CVD) and mortality due to CVD. Other atherogenic vascular diseases like stroke, pulmonary embolism and peripheral vascular diseases also pose a higher risk because of metabolic syndrome associated with psoriasis. Hyperlipidemia in psoriasis is well known because of two factors, because of psoriasis per se or because of drugs used in its treatment (cyclosporine and oral retinoids) [2]. Various cytokines like interferon $\gamma$, Tumor Necrosis Factor (TNF- $\alpha$ ), interleukins (IL)-17 and IL-22 are other markers of inflammatory process in psoriasis. IL-6, IL-17 and other chemokines are responsible for systemic inflammatory reaction in psoriasis. IL-17 causes activation of vascular endothelium leading to inflammation, atherosclerosis and thrombosis. Now the emerging concern is between psoriasis and thrombosis [3].

Psoriatic patients have higher risk of cardiovascular diseases (Myocardial Infarction, Deep Vein Thrombosis) and decreased life span [4]. This association may be due to over-representation of CVD comorbidities and mortality [5]. Underlying mechanism of these morbidities and mortality in these patients may be hyperhomocysteinemia, increased C-reactive protein (CRP), platelet hyper-reactivity and other proinflammatory cytokines [6-9]. Moreover disease related quality of life in psoriasis may lead to unhealthy life style leading to obesity (because of decreased physical activity), alcoholism and smoking which are independent risk factors for CVD [10]. Raised homocysteine further aggravates this process and is a potential marker in cardiovascular diseases.

Hyperhomocysteinemia has been found in psoriatic patients which is a marker of impending myocardial infarction, stroke, peripheral vessel diseases and pulmonary embolism. Homocysteine is a marker of hypercoagulability and atherosclerosis as well. Psoriasis patients have been reported to have higher level of homocysteine and lower level of folic acid (because of increased utilization of folic acid in psoriatic scales or its decreased absorption from intestine because of gut inflammation or because of drugs, like methotrexate) [811]. Homocysteine is produced by demethylation of methionine. Folic acid and vitamin $B_{12}$ affects the homocysteine metabolism as both of these vitamins are co-factors in DNA methylation which converts homocysteine back to methionine $[12,13]$. So decreased levels of these two vitamins are inversely proportional to homocysteine level in blood and this may cause hyperhomocysteinemia. Another pathway of homocysteine is its conversion to cystathionine. It is highly possible that hyperhomocysteinemia is responsible for cardiovascular morbidity and mortality in psoriasis because of oxidative damage to vascular endothelium.

\section{Materials and Methods}

This case-control study was conducted in dermatology outpatient department of Maharaja Agrasen Medical College (MAMC), Agroha, India in the year 2018 between September to November. Approval was taken from the ethical committee of the institution.

Thirty patients who were clinically diagnosed as chronic plaque psoriasis, attending our outpatient dermatology department, and thirty healthy, age and gender-matched controls were included in the study. Participants' age, gender, disease duration, body mass index (BMI) was recorded in a standard proforma and an informed signed consent was taken from all. Severity of the disease was calculated with standard Psoriasis Area Severity Index (PASI) score and Body Surface Area (BSA) with rule of nine.

Patients with age more than 18 years and disease duration of more than 6 months were included in our study. Patients with BMI $>30$, smokers, alcoholics, pregnant mothers, any chronic illness, cancer, hyperuricemia, taking medications affecting blood levels of homocysteine (like thiazides, theophylline, oral vitamin supplements, corticosteroids, methotrexate), were excluded from the study.

A $5 \mathrm{ml}$ fasting venous blood sample was taken and sent to the laboratory. Serum levels of homocysteine were measured by chemiluminescent immunoassay (CLIA). Values $>15.4 \mu \mathrm{mol} / \mathrm{l}$ were considered as higher levels of homocysteine.

SPSS (Statistical package for Social Science) version 17.0 software was used to analyze the data. Student's ttest of independence was applied for comparing the continuous variables for cases and controls and Chi square test of significance was used to compare the categorical variables. Pearson's correlation coefficient (r) was used for correlation of continuous variables. In all assessments for statistical significances, level of significance was confidence interval at 95\% level of confidence $(P$ value $<0.05)$. 


\section{Clinical Dermatology Open Access Journal}

\section{Results}

The mean age of psoriasis patients was $35.933 \pm 11.791$ years whereas it was $37.200 \pm 10.649$ years in control group. Both groups were identical regarding sex ratio and BMI (Table 1).

The mean duration of disease was $7.033 \pm 6.135$ years. The mean BSA involved and PASI score were $33.633 \pm 21.909$ and $16.840 \pm 10.068$.
Serum homocysteine levels were significantly higher in psoriasis patients compared to control group (Table 2).

There was positive correlation between serum homocysteine levels and BSA $(\mathrm{r}=0.397, \mathrm{p}=0.030)$ as well as PASI ( $\mathrm{r}=0.457, \mathrm{p}=0.011)$, but serum homocysteine levels did not correlate with duration of the disease.

\begin{tabular}{|c|c|c|c|}
\hline & Patients & Controls & P - value \\
\cline { 1 - 3 } $\begin{array}{c}\text { Age (years) } \\
\text { (mean } \pm \text { SD) }\end{array}$ & $35.933 \pm 11.791$ & $37.200 \pm 10.649$ & \multirow{2}{*}{0.664} \\
\hline Sex & & & \multirow{2}{*}{0.573} \\
\cline { 1 - 3 } Male (n) (\%) & $22(73.3 \%)$ & $20(66.7 \%)$ & \\
\cline { 1 - 3 } Female (n) (\%) & $8(26.7 \%)$ & $10(33.3 \%)$ & \multirow{2}{*}{0.303} \\
\cline { 1 - 3 } BMI (mean \pm SD) $\mathbf{~ k g} / \mathbf{m}^{\mathbf{2}}$ & $24.360 \pm 2.812$ & $23.653 \pm 2.446$ & \\
\cline { 1 - 3 }
\end{tabular}

Table 1: Basic characteristics of psoriasis patients $(n=30)$ and controls $(n=30)$.

\begin{tabular}{|c|c|c|c|}
\hline $\begin{array}{c}\text { Homocysteine level } \\
(\boldsymbol{\mu} \mathrm{mol} / \mathbf{l})\end{array}$ & Patients & Controls & P - value \\
\cline { 1 - 3 } Mean \pm SD & $21.831 \pm 14.785$ & $15.355 \pm 7.884$ & \multirow{2}{*}{$0.039^{*}$} \\
\cline { 1 - 3 } Minimum & 7.20 & 6.76 & \\
\hline Maximum & 68.45 & 42.20 & \\
\hline
\end{tabular}

${ }^{*} \mathrm{p}<0.05$; Significant

Table 2: Serum Homocysteine levels in psoriasis patients $(n=30)$ and controls $(n=30)$.

\section{Discussion}

The concept of homocysteine causing atherosclerosis was introduced by Mccully in 1999. Homocysteine is known to cause endothelial dysfunction leading to increased risk of thrombosis and cytotoxic effect on endothelium [14]. There could be another mechanism, DNA hypomethylation, by which homocysteine can cause atherogenesis [15]. Apart from representing a risk factor for CVD, homocysteine also plays a role in onset and progression of tissue damage underlying the disease [16]. Homocysteine and its derivatives also cause T-cell activation or T-cell apoptosis and proliferation of mononuclear cells in blood [17]. Homocysteine is known to elevate inflammatory markers, thereby triggering or aggravating inflammatory disease [16]. Hyperhomocysteinemia is an independent risk factor for arterial and venous thrombosis [18].
Psoriasis is a chronic inflammatory disease and is now considered as a systemic inflammatory disease with high incidence of dyslipidemia, low vitamin-D level, and increased homocysteine level thereby increasing cardiovascular mortality and morbidity. The incidence of myocardial infarction, thrombosis and pulmonary embolism is high in psoriasis. High serum level of homocysteine is one of the markers of cardiovascular diseases. Various studies have shown hyperhomocysteinemia in psoriasis $[8,19]$.

In our study, the mean serum homocysteine in psoriasis patients was higher than in control group and the level of homocysteine was found to be proportional to PASI score. Several studies have shown correlation of homocysteine level with PASI score whereas some studies have shown no correlation. Homocysteine level is inversely related to serum folic acid level and serum vitamin $B_{12}$ level. Though there are few reports showing 
this correlation but many studies have shown raised serum homocysteine level has no correlation with serum folic acid and vitamin $B_{12}$ (both vitamins being normal in their studies). Raised homocysteine level in psoriasis is related to low folate level which in turn is probably because of increased loss through psoriatic scales and reduced absorption from gut endothelium, which is inflamed as a consequence of systemic inflammatory process of psoriasis [20]. Folic acid is needed in demethylation of homocysteine to methionine. But many reports acknowledged normal levels of folate and vitamin $\mathrm{B}_{12}$ in psoriasis which cannot explain increased level of homocysteine in psoriasis [13].

M. Uslu et al have supported the above explanation i.e. low serum folic acid (though in their study, serum folic acid was found to be normal because of good diet, but why homocysteine was raised, they could not explain). This could be because of different DNA methylation genes for hyperhomocysteinemia [21].

Important consideration in psoriasis is that there is high incidence of cardiovascular mortality and morbidity in these patients. Higher homocysteine level (already known as a marker for CVD) can be a precipitating or aggravating factor in cardiovascular diseases, thereby increasing mortality and morbidity. Psoriasis is already associated with other metabolic disease markers like dyslipidemia. So the risk of mortality and morbidity is compounded with increased homocysteine level (which is atherogenic and thrombotic). Our suggestion is that psoriasis should be managed as a systemic inflammatory disease with a high potential of cardiovascular diseases. Chemokines and other mediators of psoriasis per se are known to cause inflammation in systemic organs. Liver disease (Non-alcoholic fatly liver disease - NAFLD through hepatocytes' inflammation), obesity (as a result of adipocyte inflammation), insulin resistance and diabetes, CVD, hypertension because of vascular involvement or renal involvement, psychiatric manifestations, mainly depression and suicidation, have all been attributed to psoriasis and chemokines (especially IL-17 and IL-22) released in systemic circulation. The inhibition of these chemokines as a part of treatment of psoriasis have been found to be very favorable in regression of all these co-morbidities.

\section{Conclusion}

Various parameters like BMI, blood pressure, lipid levels, vitamin D level, serum homocysteine and life style (smoking, alcohol, and sedentary life) should be considered while treating psoriasis patients and we should choose a proper systemic therapy which may not affect lipids in dyslipidemia patients, should correct vitamin $\mathrm{D}$ deficiency, encourage them to have a healthy life style, and screen and watch cardiovascular diseases during follow-ups. We recommend a holistic approach for the management of psoriasis to prevent co-morbidities in later stages. Scaly plaques need treatment to improve not only quality of life but also to prevent mortality and morbidity from associated or impending cardiovascular diseases and other systemic diseases associated with psoriasis.

\section{References}

1. Lebwohl M (2003) Psoriasis. Lancet 361(9364): 1197-1204.

2. Brenner S, Krakowski A, Levtov O, Heldenberg D, Werbin B, et al. (1975) Serum lipids in patients with psoriasis. Dermatologica 150(2): 96-102.

3. Gisondi P, Girolomoni G (2009) Psoriasis and atherothrombotic diseases: disease-specific and nondisease-specific risk factors. Semin Thromb Hemost 35(3): 313-324.

4. Mallbris L, Akre O, Granath F, Yin L, Lindelöf B, et al. (2004) Increased risk for cardiovascular mortality in psoriasis inpatients but not in outpatients. Eur J Epidemiol 19(3): 225-230.

5. Gelfand JM, Neimann AL, Shin DB, Wang X, Margolis DJ, et al. (2006) Risk of myocardial infarction in patients with psoriasis. JAMA 296(14): 1735-1741.

6. Tamagawa-Mineoka R, Katoh N, Veda E, Masuda K, Kishimoto S (2008) Elevated platelet activation in patients with atopic dermatitis and psoriasis: increased plasma levels of thromboglobulin and platelet factor 4. Allergol Int 57(4): 391-396.

7. Malerba M, Gisondi P, Radaeli A, Girolomoni G (2007) Psoriasis and risk of myocardial infarction. JAMA 297(4): 361-362.

8. Malerba M, Gisondi P, Radaeli A, Sala R, Calzavara Pinton PG, et al. (2006) Plasma homocysteine and folate levels in patients with chronic plaque psoriasis. Br J Dermatol 155(6): 1165-1169.

9. Sergeant A, Makrygeorgou A, Chan WC, Thorrat A, Burden D (2008) C-reactive protein in psoriasis. Br J Dermatol 158(2): 417-419. 


\section{Clinical Dermatology Open Access Journal}

10. Nijsten T, Wakkee M (2009) Complexity of the association between psoriasis and comorbidities. J Invest Dermatol 129(7): 1601-1603.

11. Kremees MH, McEvoy MT, Dann FJ, Gabriel SE (2007) Heart disease in psoriasis. J Am Acad Dermatol 57(2): 347-354.

12. Ganguly P, Alam SF (2015) Role of homocysteine in the development of cardiovascular disease. Nutrition J 14: 6 .

13. Mandaviya PR, Stolk L, Heil SG (2014) Homocysteine and DNA methylation: a review of animal and human literature. Mol Genet Metab 113(4): 243-252.

14. Nygard O, Vollset SE, Refsum H, Brattsröm L, Ueland PM (1999) Total homocysteine and cardiovascular disease. J Intern Med 246(5): 425-454.

15. Zhou S, Zhang Z, Xu G (2014) Notable epigenetic role of hyperhomocysteinemia in atherosclerosis. Lipids Health Dis 13: 134.

16. Lazzerini PE, Capecchi PL, Selvi E, Lorenzini S, Bisogno S, et al. (2007) Hyperhomocysteinemia, inflammation and autoimmunity. Autoimmun Rev 6(7): 503-509.
17. Schroecksnadel K, Frick B, Wirleitner B, Schennach H, Fuchs D (2003) Homocysteine accumulates in supernatants of stimulated human peripheral blood mononuclear cells. Clin Exp Immunol 134(1): 53-56.

18. Eldibany MM, Caprini JA (2007) Hyperhomocysteinemia and thrombosis: an overview. Arch Pathol Lab Med 131(6): 872-884.

19. Vanizor Kural B, Orem A, Cimit G, Uydu HA, Yandi YE, et al. (2003) Plasma homocysteine and its relationships with atherothrombotic markers in psoriatic patients. Clin Chim Acta 332(1-2): 23-30.

20. Scarpa R, Manguso F, D'Arienzo A, D'Armiento FP, Astarita C, et al. (2000) Microscopic inflammatory changes in colon of patients with both active psoriasis and psoriatic arthritis without bowel symptoms. J Rheumatol 27(5): 1241-1246.

21. Uslu M, Sendur N, Savk E, Karul A, Kozaci D, et al (2017) Blood homocysteine, folic acid, vitamin B12 and vitamin B6 levels in psoriasis patients. Turkderm-Turk Arch Dermatol Venereology 51: 9297. 